\title{
Identifying the occurrence time of an impending mainshock: a very recent case
}

\author{
Panayiotis A. Varotsos - Nicholas V. Sarlis • Efthimios S. Skordas • \\ Stavros-Richard G. Christopoulos $\cdot$ Mary S. Lazaridou-Varotsos
}

Received: 16 February 2015/ Accepted: 14 May 2015/Published online: 16 June 2015

(C) The Author(s) 2015. This article is published with open access at Springerlink.com

\begin{abstract}
The procedure by means of which the occurrence time of an impending mainshock can be identified by analyzing in natural time the seismicity in the candidate area subsequent to the recording of a precursory seismic electric signals (SES) activity is reviewed. Here, we report the application of this procedure to an $M_{\mathrm{W}} 5.4$ mainshock that occurred in Greece on 17 November 2014. This mainshock (which is pretty rare since it is the strongest in that area for more than half a century) was preceded by an SES activity recorded on 27 July 2014, and the results of the natural time analysis reveal that the system approached the critical point (mainshock occurrence) early in the morning on 15 November 2014.
\end{abstract}

Keywords Seismic electric signals $\cdot$ Natural time analysis - Earthquake prediction - Critical phenomena

\section{Introduction}

Earthquakes (EQs) in general exhibit complex correlations in time, space, and magnitude $M$, which have been investigated by several authors (Sornette 2000; Corral 2004; Davidsen and Paczuski 2005; Holliday et al. 2006; Saichev and Sornette 2006; Eichner et al. 2007; Lennartz et al. 2008, 2011; Lippiello et al. 2009, 2012; Sarlis et al. 2009, 2010; Telesca and Lovallo 2009; Bottiglieri et al. 2010; Telesca 2010; Sarlis 2011; Huang and Ding 2012; Sarlis

P. A. Varotsos $(\bowtie) \cdot$ N. V. Sarlis · E. S. Skordas ·

S.-R. G. Christopoulos · M. S. Lazaridou-Varotsos

Department of Solid State Physics and Solid Earth Physics

Institute, Faculty of Physics, School of Science, National and

Kapodistrian University of Athens, Panepistimiopolis, Zografos,

15784 Athens, Greece

e-mail: pvaro@otenet.gr and Christopoulos 2012; Varotsos et al. 2011c, 2012). The observed earthquake scaling laws (Turcotte 1997) indicate the existence of phenomena closely associated with the proximity of the system to a critical point, e.g., see Holliday et al. (2006), since scaling is a hallmark of criticality (Stanley 1999). Here, we take this view that mainshocks are (non-equilibrium) critical phenomena.

Major EQs are preceded by transient changes of the electric field of the Earth termed seismic electric signals (SES) (Varotsos and Alexopoulos 1984a, b). A series of such signals recorded within a short time are called SES activities (Varotsos and Lazaridou 1991; Varotsos et al. 1993, 2009; Varotsos 2005), the average leading time of which is of the order of a few months (Varotsos et al. 2011a). It has been suggested that SES are emitted when the stress in the focal area of the impending mainshock reaches a critical value (Varotsos and Alexopoulos 1984a, b, 1986; Varotsos et al. 2011b). This suggestion is strengthened by the recent finding (Varotsos et al. 2013) that the fluctuations of the order parameter of seismicity defined in the frame of natural time analysis (see the next section) minimize upon the initiation of an SES activity exhibiting long-range temporal correlations (Varotsos et al. 2014). Such minima of the fluctuations of the order parameter of seismicity have been identified before all major $(M \geq 7.6) \mathrm{EQs}$ in Japan (Sarlis et al. 2013, 2015).

The identification of the occurrence time of an impending mainshock within a short time window is a challenge. This becomes possible when employing a procedure that combines SES data and natural time analysis of the seismicity (Varotsos et al. 2001, 2002a, b, 2005, 2008, 2011a; Sarlis et al. 2008; Sarlis 2013). In short, the initiation of the SES activity marks the time when the system enters the critical stage, and then the natural time analysis of the subsequent seismicity in the candidate area (which is 
determined on the basis of SES data, e.g., see Varotsos 2005) identifies when the system approaches the critical point, i.e., the mainshock occurrence, e.g., see Fig. 1 of Huang (2015). It is the scope of this paper to report a characteristic application of this procedure, which refers to an SES activity that was followed by a pronounced $M_{\mathrm{W}} 5.4$ mainshock in Greece on 17 November 2014, which is pretty rare as explained later.

\section{Summary of the procedure to identify the occurrence time of an impending mainshock}

Let us first summarize the natural time analysis (Varotsos et al. 2002a) in the case of seismicity: In a time series comprising $N$ EQs, the natural time $\chi_{k}=k / N$ serves as an index for the occurrence of the $k$-th EQ. The combination of this index with the energy $Q_{k}$ released during the $k$-th EQ of magnitude $M_{k}$, i.e., the pair $\left(\chi_{k}, Q_{k}\right)$, is studied in natural time analysis. Alternatively, one studies the pair $\left(\chi_{k}, p_{k}\right)$, where $p_{k}=Q_{k} / \sum_{n=1}^{N} Q_{n}$ stands for the normalized energy released during the $k$-th EQ. It has been found that the variance of $\chi$ weighted for $p_{k}$, designated by $\kappa_{1}$, which is given by (Varotsos et al. 2001, 2002a, b, 2003a, b, 2011a):

$\kappa_{1}=\left\langle\chi^{2}\right\rangle-\langle\chi\rangle^{2}=\sum_{k=1}^{N} p_{k} \chi_{k}^{2}-\left(\sum_{k=1}^{N} p_{k} \chi_{k}\right)^{2}$

plays a prominent role in natural time analysis. In particular, $\kappa_{1}$ may serve as an order parameter for seismicity

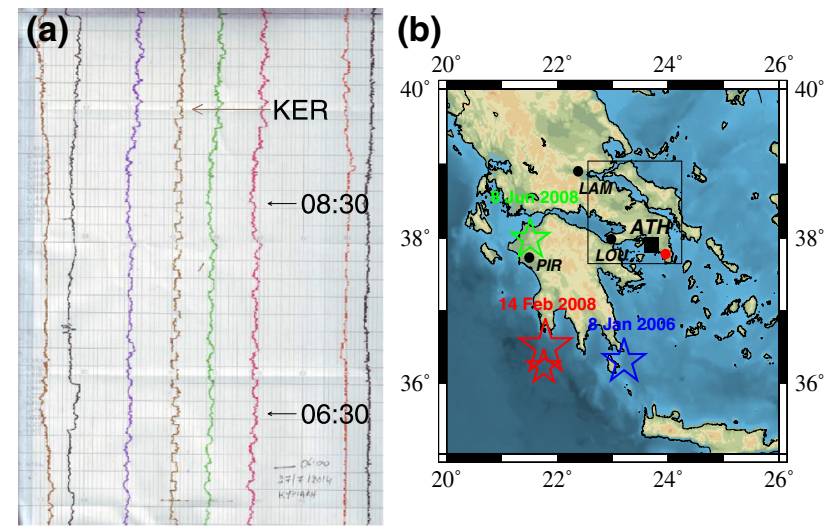

Fig. 1 a The SES activity of dichotomous nature recorded at the Keratea (KER) geoelectrical station of the SES telemetric network. bThe predicted epicentral area designated by the rectangle on a map in which the location of the KER station (red bullet) is shown along with that of other geoelectrical stations Lamia (LAM), Loutraki (LOU) and Pirgos (PIR) (black bullets). The epicenters of the strongest $\mathrm{EQs}$ in Greece $\left(M_{\mathrm{W}} \geq 6.5\right)$ during the last decade are also shown with stars. The central station of the SES telemetric network is located at Athens (ATH, black square)
(Varotsos et al. 2005), and it has been empirically observed (Varotsos et al. 2001, 2002b, 2005, 2008, 2011a; Sarlis et al. 2008; Sarlis 2013) that $\kappa_{1}$ of the seismicity in the candidate area when considering all EQs with magnitude equal to or larger than a magnitude threshold $M_{\text {thres }}$ subsequent to an SES activity becomes equal to 0.070 when approaching the critical point (mainshock occurrence). Note that $Q_{k}$, and hence $p_{k}$, for EQs is estimated through the usual relation (Kanamori 1978): $Q_{k} \propto 10^{1.5 M_{k}}$.

Upon the recording of an SES activity, one can estimate an area $A$ within which the impending mainshock is expected to occur. The magnitude $M$ of the expected EQ is estimated through the relation $\log _{10}\left(\frac{\Delta V}{L}\right) \approx 0.3 M+$ const., e.g., see Varotsos and Lazaridou (1991), where for a given measuring dipole of length $L$ and a given seismic area the SES amplitude $\Delta V / L$ is found from the anomalous variation $\Delta V$ of the potential difference between the corresponding two electrodes. When area $A$ reaches criticality, one expects in general that all its subareas have also reached criticality simultaneously. At that time, therefore, the evolution of seismicity in each of its subareas is expected to result in $\kappa_{1}$ values close to 0.070 . Assuming equipartition of probability among the subareas (Sarlis et al. 2008), the distribution $\operatorname{Prob}\left(\kappa_{1}\right)$ of the $\kappa_{1}$ values of all subareas should be peaked at around 0.070 exhibiting also magnitude threshold invariance. This usually occurs a few days to around 1 week before the mainshock, thus it enables the prediction of the occurrence time of major EQs with time window of the order of a week or less.

\section{Application to a recent pronounced seismic activity in Greece}

The SES activity shown in Fig. 1a was recorded on 27 July 2014 at Keratea (KER) geoelectrical station, the location of which is depicted with the red bullet in Fig. 1b. On the basis of the selectivity map of this station [i.e., the map showing all seismic areas in the past that gave rise to SES recorded at this station, e.g., see Varotsos and Lazaridou (1991)] and the ratio of the SES components, the candidate area was determined (Sarlis et al. 2014). This is depicted here by the rectangle in Fig. $1 \mathrm{~b}$ as was designated in the uppermost right part of Fig. 2 of the paper uploaded by Sarlis et al. (2014) on 7 August 2014.

We now proceed to the natural time analysis of the seismicity subsequent to the aforementioned SES activity at KER within the candidate area $37.7^{\circ} \mathrm{N}-39.0^{\circ} \mathrm{N}, 22.6^{\circ} \mathrm{E}-$ $24.2^{\circ} \mathrm{E}$. The EQ catalog of the Institute of Geodynamics of the National Observatory of Athens available on 2 February 2015 at http://www.gein.noa.gr/services/current_cata logue.php was used, e.g., see Chouliaras et al. (2013); Mignan and Chouliaras (2014). Figure 2a depicts $\operatorname{Prob}\left(\kappa_{1}\right)$ 

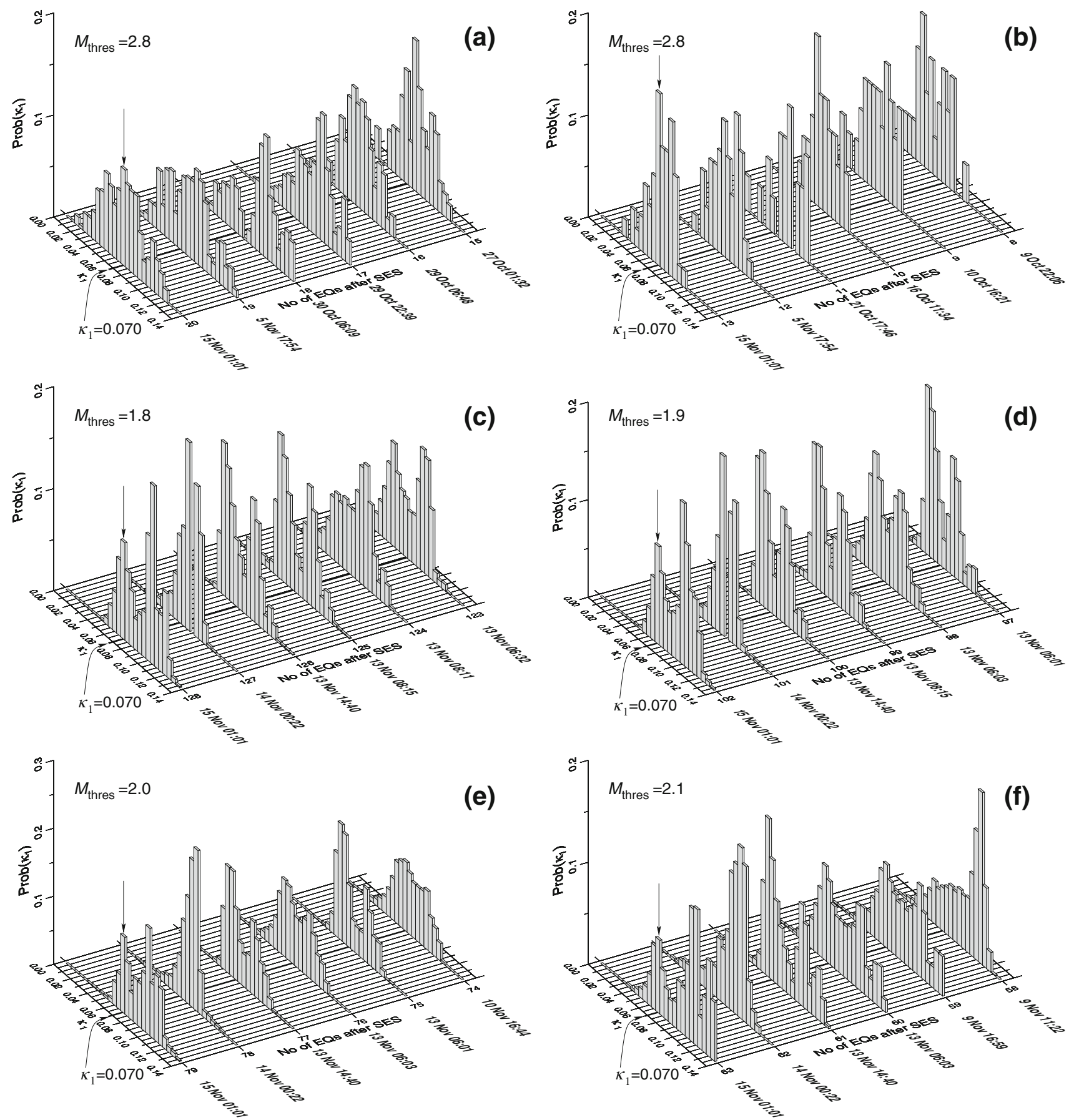

Fig. 2 How the histograms of $\operatorname{Prob}\left(\kappa_{1}\right)$ versus $\kappa_{1}$ evolve event by event in the natural time analysis of the seismicity subsequent to the initiation of the SES activity depicted in Fig. 1a. In each panel, the magnitude threshold ( $\left.M_{\text {thres }}\right)$ used in the calculation is also depicted. For details on the exact (sub) areas within the rectangle of Fig. 1b considered in each panel, see Sect. 3

versus $\kappa_{1}$ of seismicity for $M_{\text {thres }}=2.8$ (the data used are compiled in Table 1) for the period after 27 October 2014, i.e., almost three weeks before the mainshock occurrence on 17 November 2014. During this period, six smaller EQs occurred and we observe that $\operatorname{Prob}\left(\kappa_{1}\right)$ maximizes at $\kappa_{1}=0.070$ upon the occurrence of the last EQ, i.e., the $M_{\mathrm{L}}$
2.8 EQ at 01:01 UT on 15 November 2014. It is remarkable that the same behavior is observed in Fig. 2b, where in the computation of the $\kappa_{1}$ values, we discarded from the seismicity of the candidate area $37.7^{\circ} \mathrm{N}-39.0^{\circ} \mathrm{N}, 22.6^{\circ} \mathrm{E}-$ $24.2^{\circ} \mathrm{E}$ the EQs that occurred within the subarea $37.7^{\circ} \mathrm{N}-$ $38.3^{\circ} \mathrm{N}, 22.6^{\circ} \mathrm{E}-23.3^{\circ} \mathrm{E}$. This is consistent with the fact that 
the latter subarea constitutes the preliminary selectivity map of the LOU station, see Fig. 1b, which however did not show any SES activity simultaneously with the one initiated on 27 July 2014 at KER station (alternatively, the area resulting from the subtraction of the above two areas could have been announced as a candidate area for the
Table 1 All EQs with

$M_{\mathrm{L}} \geq 2.8$ that occurred after the initiation of the SES activity on 27 July 2014 within the area $37.7^{\circ} \mathrm{N}-39.0^{\circ} \mathrm{N}, 22.6^{\circ} \mathrm{E}-24.2^{\circ} \mathrm{E}$, as they were reported on 2 February 2015 by the Institute of Geodynamics of the National Observatory of Athens
Table 2 All EQs with

$M_{\mathrm{L}} \geq 1.8$ that occurred after the initiation of the SES activity on 27 July 2014 within the area $37.7^{\circ} \mathrm{N}-39.0^{\circ} \mathrm{N}, 22.6^{\circ} \mathrm{E}-24.2^{\circ} \mathrm{E}$ excluding those (see the text) within the subarea $37.7^{\circ} \mathrm{N}-$ $38.3^{\circ} \mathrm{N}, 22.6^{\circ} \mathrm{E}-23.3^{\circ} \mathrm{E}$, as they were reported on 2 February 2015 by the Institute of Geodynamics of the National Observatory of Athens

\begin{tabular}{|c|c|c|c|c|c|}
\hline \multicolumn{2}{|l|}{ Orignal time } & \multirow[t]{2}{*}{ Lat. $\left({ }^{\circ} \mathrm{N}\right)$} & \multirow[t]{2}{*}{ Long. $\left({ }^{\circ} \mathrm{E}\right)$} & \multirow[t]{2}{*}{ Depth (km) } & \multirow[t]{2}{*}{$M_{\mathrm{L}}$} \\
\hline Date (a-mo-d) & Time (h:min:s) & & & & \\
\hline 2014-07-30 & 00:55:04 & 38.04 & 24.12 & 19 & 3.6 \\
\hline 2014-08-01 & $22: 36: 46$ & 38.95 & 24.15 & 24 & 3.0 \\
\hline 2014-08-02 & 23:00:04 & 38.04 & 24.11 & 14 & 3.0 \\
\hline 2014-08-08 & $00: 29: 42$ & 38.92 & 23.10 & 19 & 2.9 \\
\hline 2014-08-08 & $00: 30: 23$ & 38.92 & 23.09 & 14 & 3.4 \\
\hline $2014-08-25$ & 03:40:13 & 38.09 & 22.74 & 15 & 2.9 \\
\hline 2014-09-18 & $07: 24: 25$ & 37.70 & 23.09 & 25 & 3.6 \\
\hline 2014-09-24 & $16: 05: 31$ & 38.73 & 22.58 & 11 & 2.8 \\
\hline 2014-10-06 & 02:29:51 & 38.96 & 23.22 & 21 & 2.8 \\
\hline 2014-10-09 & $22: 06: 28$ & 38.25 & 24.11 & 18 & 2.8 \\
\hline 2014-10-10 & $16: 21: 09$ & 38.80 & 23.10 & 11 & 2.8 \\
\hline 2014-10-11 & $01: 53: 35$ & 37.88 & 22.55 & 19 & 3.2 \\
\hline 2014-10-16 & $11: 34: 17$ & 38.75 & 23.38 & 24 & 2.8 \\
\hline 2014-10-21 & $17: 46: 25$ & 38.59 & 22.99 & 23 & 3.2 \\
\hline $2014-10-27$ & $01: 32: 24$ & 38.14 & 22.63 & 14 & 3.1 \\
\hline 2014-10-29 & $06: 48: 12$ & 38.13 & 22.63 & 17 & 3.1 \\
\hline 2014-10-29 & $22: 39: 37$ & 38.14 & 22.64 & 15 & 3.4 \\
\hline 2014-10-30 & 06:09:08 & 38.14 & 22.63 & 15 & 3.7 \\
\hline 2014-11-05 & $17: 54: 39$ & 38.65 & 23.95 & 24 & 3.0 \\
\hline 2014-11-15 & 01:01:23 & 37.79 & 23.96 & 186 & 2.8 \\
\hline 2014-11-15 & 08:11:31 & 38.99 & 23.70 & 24 & 3.7 \\
\hline 2014-11-17 & 23:05:55 & 38.64 & 23.40 & 24 & 5.2 \\
\hline 2014-11-17 & 23:09:03 & 38.64 & 23.41 & 23 & 5.2 \\
\hline
\end{tabular}

\begin{tabular}{llllll}
\hline Orignal time & & Lat. $\left({ }^{\circ} \mathrm{N}\right)$ & Long. $\left({ }^{\circ} \mathrm{E}\right)$ & Depth $(\mathrm{km})$ & $M_{\mathrm{L}}$ \\
\hline Date (a-mo-d) & Time $(\mathrm{h}: \mathrm{min}: \mathrm{s})$ & & & & \\
\hline $2014-07-30$ & $00: 55: 04$ & 38.04 & 24.12 & 19 & 3.6 \\
$2014-07-30$ & $09: 14: 12$ & 38.82 & 23.30 & 22 & 2.1 \\
$2014-07-31$ & $04: 18: 24$ & 38.93 & 24.12 & 12 & 1.8 \\
$2014-07-31$ & $08: 02: 16$ & 38.14 & 23.52 & 10 & 1.8 \\
$2014-07-31$ & $12: 22: 06$ & 38.66 & 23.58 & 3 & 1.8 \\
$2014-07-31$ & $15: 12: 24$ & 38.58 & 23.58 & 16 & 2.2 \\
$2014-08-01$ & $01: 46: 54$ & 38.72 & 22.74 & 13 & 1.9 \\
$2014-08-01$ & $21: 06: 38$ & 38.89 & 23.53 & 17 & 1.9 \\
$2014-08-01$ & $22: 32: 32$ & 38.94 & 24.13 & 11 & 2.2 \\
$2014-08-01$ & $22: 36: 46$ & 38.95 & 24.15 & 24 & 3.0 \\
$2014-08-02$ & $07: 32: 40$ & 38.94 & 24.12 & 16 & 1.9 \\
$2014-08-02$ & $23: 00: 41$ & 38.04 & 24.11 & 14 & 3.0 \\
$2014-08-02$ & $23: 02: 36$ & 38.04 & 24.11 & 11 & 2.6 \\
$2014-08-05$ & $16: 18: 27$ & 38.74 & 22.57 & 10 & 1.9 \\
$2014-08-07$ & $03: 38: 12$ & 38.27 & 24.13 & 20 & 2.7 \\
$2014-08-07$ & $06: 00: 09$ & 38.26 & 24.15 & 11 & 2.2 \\
\hline
\end{tabular}


Table 2 continued

\begin{tabular}{|c|c|c|c|c|c|}
\hline \multicolumn{2}{|l|}{ Orignal time } & \multirow[t]{2}{*}{ Lat. $\left({ }^{\circ} \mathrm{N}\right)$} & \multirow[t]{2}{*}{ Long. $\left({ }^{\circ} \mathrm{E}\right)$} & \multirow[t]{2}{*}{ Depth $(\mathrm{km})$} & \multirow[t]{2}{*}{$M_{\mathrm{L}}$} \\
\hline Date (a-mo-d) & Time (h:min:s) & & & & \\
\hline 2014-08-07 & 06:09:57 & 38.27 & 24.14 & 11 & 1.9 \\
\hline 2014-08-07 & 13:44:07 & 38.76 & 23.87 & 13 & 1.9 \\
\hline 2014-08-07 & $20: 28: 32$ & 38.27 & 24.16 & 22 & 2.6 \\
\hline 2014-08-08 & $00: 29: 42$ & 38.92 & 23.10 & 19 & 2.9 \\
\hline 2014-08-08 & $00: 30: 23$ & 38.92 & 23.09 & 14 & 3.4 \\
\hline 2014-08-08 & $00: 36: 13$ & 38.92 & 23.09 & 11 & 1.8 \\
\hline 2014-08-08 & $00: 36: 59$ & 38.92 & 23.09 & 13 & 1.8 \\
\hline 2014-08-08 & 01:13:03 & 38.91 & 23.10 & 16 & 2.2 \\
\hline 2014-08-08 & $12: 03: 30$ & 38.76 & 23.48 & 11 & 1.8 \\
\hline 2014-08-08 & $14: 05: 13$ & 38.26 & 24.15 & 12 & 2.5 \\
\hline 2014-08-08 & 14:06:05 & 38.26 & 24.14 & 12 & 2.3 \\
\hline 2014-08-08 & 14:28:59 & 38.27 & 24.15 & 12 & 2.2 \\
\hline 2014-08-08 & 17:03:06 & 38.38 & 23.97 & 15 & 2.3 \\
\hline 2014-08-10 & 01:54:07 & 38.90 & 23.06 & 19 & 2.3 \\
\hline 2014-08-10 & 07:31:10 & 38.27 & 24.13 & 22 & 2.4 \\
\hline 2014-08-14 & $05: 31: 15$ & 38.70 & 22.82 & 13 & 2.6 \\
\hline 2014-08-14 & 08:51:11 & 38.56 & 23.64 & 18 & 1.8 \\
\hline 2014-08-14 & 11:43:06 & 38.71 & 22.79 & 10 & 2.1 \\
\hline 2014-08-14 & $15: 13: 27$ & 38.56 & 23.28 & 20 & 1.8 \\
\hline 2014-08-15 & 09:56:59 & 38.80 & 23.52 & 25 & 1.8 \\
\hline 2014-08-20 & $21: 44: 19$ & 38.67 & 22.63 & 18 & 2.3 \\
\hline 2014-08-23 & $16: 26: 48$ & 38.55 & 24.11 & 15 & 2.2 \\
\hline 2014-08-26 & $07: 45: 48$ & 38.15 & 23.52 & 11 & 1.9 \\
\hline 2014-08-26 & $16: 39: 17$ & 38.74 & 22.76 & 10 & 2.6 \\
\hline 2014-08-28 & 18:00:27 & 38.74 & 22.76 & 7 & 1.9 \\
\hline 2014-08-31 & 09:16:52 & 38.95 & 23.02 & 15 & 1.9 \\
\hline 2014-09-01 & 15:00:55 & 38.87 & 23.57 & 16 & 1.9 \\
\hline 2014-09-02 & $11: 48: 54$ & 37.79 & 23.86 & 13 & 2.1 \\
\hline 2014-09-02 & 13:00:44 & 38.88 & 23.57 & 25 & 2.3 \\
\hline 2014-09-02 & $21: 33: 56$ & 38.72 & 22.80 & 12 & 2.5 \\
\hline 2014-09-03 & $15: 10: 55$ & 38.60 & 22.66 & 19 & 1.8 \\
\hline 2014-09-03 & $16: 56: 34$ & 38.45 & 23.72 & 15 & 1.9 \\
\hline 2014-09-05 & $00: 46: 08$ & 38.47 & 22.56 & 18 & 1.8 \\
\hline 2014-09-06 & $02: 49: 22$ & 38.82 & 23.12 & 11 & 1.8 \\
\hline 2014-09-06 & $23: 40: 45$ & 38.88 & 24.14 & 7 & 1.8 \\
\hline 2014-09-07 & $05: 47: 43$ & 37.80 & 23.87 & 9 & 2.3 \\
\hline 2014-09-07 & 06:05:55 & 39.02 & 23.53 & 25 & 2.3 \\
\hline 2014-09-08 & 08:46:39 & 38.73 & 22.57 & 17 & 2.1 \\
\hline 2014-09-08 & $16: 05: 48$ & 38.82 & 23.12 & 13 & 2.2 \\
\hline 2014-09-10 & $16: 51: 54$ & 38.71 & 22.73 & 21 & 2.6 \\
\hline 2014-09-12 & $19: 22: 00$ & 37.80 & 23.87 & 8 & 2.6 \\
\hline 2014-09-13 & $13: 26: 51$ & 38.72 & 22.77 & 13 & 1.8 \\
\hline 2014-09-14 & $12: 40: 30$ & 38.70 & 22.74 & 16 & 2.1 \\
\hline 2014-09-14 & $16: 11: 42$ & 38.23 & 24.05 & 19 & 2.0 \\
\hline 2014-09-14 & $16: 35: 40$ & 37.89 & 23.58 & 15 & 2.7 \\
\hline 2014-09-15 & 05:05:09 & 38.73 & 23.92 & 22 & 2.5 \\
\hline 2014-09-15 & 07:09:11 & 37.89 & 23.55 & 15 & 1.9 \\
\hline 2014-09-16 & $15: 32: 53$ & 38.76 & 23.94 & 17 & 2.0 \\
\hline
\end{tabular}


Table 2 continued

\begin{tabular}{|c|c|c|c|c|c|}
\hline \multicolumn{2}{|l|}{ Orignal time } & \multirow[t]{2}{*}{ Lat. $\left({ }^{\circ} \mathrm{N}\right)$} & \multirow[t]{2}{*}{ Long. $\left({ }^{\circ} \mathrm{E}\right)$} & \multirow[t]{2}{*}{ Depth $(\mathrm{km})$} & \multirow[t]{2}{*}{$M_{\mathrm{L}}$} \\
\hline Date (a-mo-d) & Time (h:min:s) & & & & \\
\hline 2014-09-17 & $07: 40: 18$ & 38.58 & 23.20 & 13 & 2.2 \\
\hline 2014-09-20 & 19:18:57 & 39.03 & 23.29 & 15 & 1.9 \\
\hline 2014-09-22 & 03:00:50 & 38.73 & 22.59 & 11 & 2.0 \\
\hline 2014-09-23 & 01:51:40 & 38.36 & 23.80 & 19 & 2.1 \\
\hline 2014-09-24 & $16: 05: 31$ & 38.73 & 22.58 & 11 & 2.8 \\
\hline 2014-09-24 & $16: 29: 10$ & 38.72 & 22.74 & 12 & 1.8 \\
\hline 2014-09-24 & $16: 33: 38$ & 38.73 & 23.91 & 11 & 2.1 \\
\hline 2014-09-25 & 03:07:58 & 38.67 & 23.08 & 12 & 2.1 \\
\hline 2014-09-27 & $05: 25: 33$ & 38.73 & 23.91 & 15 & 2.5 \\
\hline 2014-09-29 & 00:16:07 & 38.99 & 23.24 & 16 & 1.8 \\
\hline 2014-09-30 & 09:00:08 & 38.12 & 23.52 & 9 & 1.8 \\
\hline 2014-09-30 & $10: 17: 15$ & 37.88 & 23.66 & 12 & 2.0 \\
\hline 2014-10-01 & 08:31:01 & 38.93 & 24.07 & 15 & 1.9 \\
\hline 2014-10-02 & 06:00:33 & 37.80 & 23.88 & 9 & 2.0 \\
\hline 2014-10-04 & $04: 43: 17$ & 38.96 & 23.21 & 14 & 1.9 \\
\hline 2014-10-06 & 02:29:51 & 38.96 & 23.22 & 21 & 2.8 \\
\hline 2014-10-06 & 15:39:03 & 38.71 & 22.79 & 12 & 2.2 \\
\hline 2014-10-06 & 18:00:10 & 39.02 & 24.07 & 15 & 2.3 \\
\hline 2014-10-07 & 09:07:14 & 38.18 & 23.53 & 11 & 1.8 \\
\hline 2014-10-08 & $18: 41: 31$ & 38.67 & 22.66 & 15 & 1.9 \\
\hline 2014-10-09 & $08: 48: 54$ & 38.15 & 23.53 & 14 & 1.8 \\
\hline 2014-10-09 & 21:31:09 & 38.95 & 24.13 & 27 & 2.6 \\
\hline 2014-10-09 & $22: 06: 28$ & 38.25 & 24.11 & 18 & 2.8 \\
\hline 2014-10-10 & 02:05:37 & 38.92 & 22.96 & 10 & 1.8 \\
\hline 2014-10-10 & $16: 21: 09$ & 38.80 & 23.10 & 11 & 2.8 \\
\hline 2014-10-11 & $14: 47: 39$ & 38.69 & 22.80 & 10 & 2.1 \\
\hline 2014-10-13 & 02:07:14 & 38.40 & 23.96 & 22 & 1.8 \\
\hline 2014-10-16 & $11: 34: 17$ & 38.75 & 23.38 & 24 & 2.8 \\
\hline 2014-10-16 & $17: 31: 39$ & 39.02 & 23.27 & 14 & 1.8 \\
\hline 2014-10-17 & 09:24:40 & 38.68 & 23.10 & 8 & 1.9 \\
\hline 2014-10-19 & 20:06:03 & 38.60 & 23.30 & 14 & 2.0 \\
\hline 2014-10-21 & 11:30:48 & 38.36 & 23.99 & 17 & 2.1 \\
\hline 2014-10-21 & $17: 46: 25$ & 38.59 & 22.99 & 23 & 3.2 \\
\hline 2014-10-21 & 18:04:26 & 38.59 & 22.97 & 15 & 2.0 \\
\hline 2014-10-24 & 00:19:54 & 38.34 & 23.54 & 14 & 1.9 \\
\hline 2014-10-25 & $17: 54: 53$ & 38.36 & 23.82 & 20 & 2.0 \\
\hline 2014-10-26 & 12:06:05 & 38.74 & 22.57 & 9 & 1.9 \\
\hline 2014-10-27 & 17:37:07 & 38.56 & 24.10 & 8 & 1.9 \\
\hline 2014-10-30 & 03:02:01 & 38.77 & 23.53 & 25 & 2.1 \\
\hline 2014-11-01 & $16: 59: 53$ & 38.71 & 22.56 & 13 & 2.0 \\
\hline 2014-11-01 & $21: 51: 13$ & 38.08 & 23.61 & 17 & 2.0 \\
\hline 2014-11-02 & 00:45:05 & 38.78 & 23.31 & 15 & 2.0 \\
\hline 2014-11-03 & 05:09:28 & 38.08 & 23.61 & 18 & 2.2 \\
\hline 2014-11-03 & $08: 28: 52$ & 39.01 & 23.33 & 21 & 1.9 \\
\hline 2014-11-05 & $16: 52: 38$ & 38.65 & 23.94 & 22 & 2.4 \\
\hline 2014-11-05 & $17: 54: 39$ & 38.65 & 23.95 & 24 & 3.0 \\
\hline 2014-11-06 & 02:52:05 & 38.81 & 23.50 & 15 & 1.8 \\
\hline 2014-11-07 & 11:58:18 & 38.64 & 23.92 & 13 & 2.0 \\
\hline
\end{tabular}


Table 2 continued

\begin{tabular}{llllll}
\hline Orignal time & & Lat. $\left({ }^{\circ} \mathrm{N}\right)$ & Long. $\left({ }^{\circ} \mathrm{E}\right)$ & Depth $(\mathrm{km})$ & $M_{\mathrm{L}}$ \\
\hline Date (a-mo-d) & Time $(\mathrm{h}: \mathrm{min}: \mathrm{s})$ & & & & \\
\hline $2014-11-08$ & $21: 00: 55$ & 38.73 & 22.58 & 7 & 2.2 \\
$2014-11-09$ & $02: 13: 07$ & 38.21 & 24.07 & 12 & 2.0 \\
$2014-11-09$ & $11: 22: 31$ & 39.02 & 23.42 & 25 & 2.3 \\
$2014-11-09$ & $16: 59: 54$ & 38.64 & 23.91 & 13 & 2.2 \\
$2014-11-10$ & $16: 44: 19$ & 38.63 & 23.92 & 12 & 2.0 \\
$2014-11-10$ & $16: 44: 42$ & 38.64 & 23.94 & 13 & 2.0 \\
$2014-11-12$ & $02: 48: 58$ & 39.04 & 23.54 & 14 & 1.9 \\
$2014-11-12$ & $19: 15: 54$ & 38.82 & 23.38 & 16 & 1.8 \\
$2014-11-13$ & $06: 01: 31$ & 38.72 & 22.58 & 10 & 2.0 \\
$2014-11-13$ & $06: 03: 04$ & 38.36 & 23.55 & 14 & 2.4 \\
$2014-11-13$ & $06: 32: 59$ & 38.87 & 23.55 & 15 & 1.8 \\
$2014-11-13$ & $08: 11: 04$ & 38.47 & 24.10 & 11 & 1.8 \\
$2014-11-13$ & $08: 15: 49$ & 38.67 & 22.67 & 18 & 1.9 \\
$2014-11-13$ & $14: 40: 13$ & 38.70 & 22.70 & 8 & 2.4 \\
$2014-11-14$ & $00: 22: 20$ & 39.00 & 24.15 & 21 & 2.1 \\
$2014-11-15$ & $01: 01: 23$ & 37.79 & 23.96 & 186 & 2.8 \\
$2014-11-15$ & $08: 11: 31$ & 38.99 & 23.70 & 24 & 3.7 \\
$2014-11-17$ & $23: 05: 55$ & 38.64 & 23.40 & 24 & 5.2 \\
$2014-11-17$ & $23: 09: 03$ & 38.64 & 23.41 & 23 & \\
\hline & & & & & \\
\hline
\end{tabular}

impending mainshock). To assure that this behavior exhibits also magnitude threshold invariance, we repeated the calculation that resulted in Fig. 2b, but for low magnitude thresholds (so that to have a large number of EQs). In particular, Fig. 2c-f depict the corresponding results for $M_{\text {thres }}=1.8,1.9,2.0$, and 2.1, respectively, which do show that $\operatorname{Prob}\left(\kappa_{1}\right)$ versus $\kappa_{1}$ exhibits local maximum at $\kappa_{1}=0.070$ upon the occurrence of the aforementioned EQ on 15 November 2014 (the seismic data used in order to obtain Fig. 2b-f are given in Table 2). Actually, almost three days later, i.e., at 23:05 UT on 17 November 2014, the $M_{\mathrm{W}}$ $(\mathrm{USGS})=5.4 \mathrm{EQ}$ occurred with an epicenter at $38.67^{\circ} \mathrm{N}$, $23.39^{\circ} \mathrm{E}$ (followed by a smaller $M_{\mathrm{W}}$ (USGS) 5.1 EQ at 23:09 UT with epicenter at $38.68^{\circ} \mathrm{N}, 23.24^{\circ} \mathrm{E}$ ). It should be mentioned that EQs of such magnitude occur there very rarely. In particular, no EQ with $M_{\mathrm{W}}$ (USGS) $\geq 5.4$ took place within the coordinates $38.3^{\circ} \mathrm{N}-39.0^{\circ} \mathrm{N}, 23.0^{\circ} \mathrm{E}-23.8^{\circ} \mathrm{E}$ since 1965 . In view of this very rare occurrence, it is interesting to study this case in the future by employing an approach (Moustra et al. 2011) which uses SES and a neural network (trained by relevant data of earlier cases) to predict the magnitude and the occurrence time of the forthcoming EQ.

\section{Conclusion}

A pronounced $M_{\mathrm{W}}$ (USGS) $=5.4 \mathrm{EQ}$ was strongly felt at Athens, Greece, on 17 November 2014. This is pretty rare since it is the strongest EQ that occurred in that area since 1965. The procedure based on natural time analysis of the seismicity subsequent to an SES activity recorded on 27 July 2014 at the KER station close to Athens revealed that the system approached the critical point (mainshock occurrence) just a few days before, i.e., on 15 November 2014.

Acknowledgments We gratefully acknowledge the continuous supervision and technical support of the geoelectrical stations of the SES telemetric network by Basil Dimitropoulos, Spyros Tzigkos and George Lampithianakis.

Financial support None.

Open Access This article is distributed under the terms of the Creative Commons Attribution 4.0 International License (http:// creativecommons.org/licenses/by/4.0/), which permits unrestricted use, distribution, and reproduction in any medium, provided you give appropriate credit to the original author(s) and the source, provide a link to the Creative Commons license, and indicate if changes were made.

\section{References}

Bottiglieri M, de Arcangelis L, Godano C, Lippiello E (2010) Multiple-time scaling and universal behavior of the earthquake interevent time distribution. Phys Rev Lett 104:158501

Chouliaras G, Melis NS, Drakatos G, Makropoulos K (2013) Operational network improvements and increased reporting in the NOA (Greece) seismicity catalog. Adv Geosci 36:7-9

Corral A (2004) Long-term clustering, scaling, and universality in the temporal occurrence of earthquakes. Phys Rev Lett 92:108501 
Davidsen J, Paczuski M (2005) Analysis of the spatial distribution between successive earthquakes. Phys Rev Lett 94:048501

Eichner JF, Kantelhardt JW, Bunde A, Havlin S (2007) Statistics of return intervals in long-term correlated records. Phys Rev E 75:011128

Holliday JR, Rundle JB, Turcotte DL, Klein W, Tiampo KF, Donnellan A (2006) Space-time clustering and correlations of major earthquakes. Phys Rev Lett 97:238501

Huang Q (2015) Forecasting the epicenter of a future major earthquake. Proc Natl Acad Sci USA 112:944-945

Huang Q, Ding X (2012) Spatiotemporal variations of seismic quiescence prior to the $2011 M 9.0$ Tohoku earthquake revealed by an improved region-time-length algorithm. Bull Seismol Soc Am 102:1878-1883

Kanamori H (1978) Quantification of earthquakes. Nature 271:411-414

Lennartz S, Livina VN, Bunde A, Havlin S (2008) Long-term memory in earthquakes and the distribution of interoccurrence times. EPL 81:69001

Lennartz S, Bunde A, Turcotte DL (2011) Modelling seismic catalogues by cascade models: do we need long-term magnitude correlations? Geophys J Int 184:1214-1222

Lippiello E, de Arcangelis L, Godano C (2009) Role of static stress diffusion in the spatiotemporal organization of aftershocks. Phys Rev Lett 103:038501

Lippiello E, Godano C, de Arcangelis L (2012) The earthquake magnitude is influenced by previous seismicity. Geophys Res Lett 39:L05309

Mignan A, Chouliaras G (2014) Fifty years of seismic network performance in Greece (1964-2013): spatiotemporal evolution of the completeness magnitude. Seismol Res Lett 85:657-667

Moustra M, Avraamides M, Christodoulou C (2011) Artificial neural networks for earthquake prediction using time series magnitude data or seismic electric signals. Expert Syst Appl 38:15032-15039

Saichev A, Sornette D (2006) "Universal" distribution of inter earthquake times explained. Phys Rev Lett 97:078501

Sarlis NV (2011) Magnitude correlations in global seismicity. Phys Rev E 84:022101

Sarlis NV (2013) On the recent seismic activity in North-Eastern Aegean Sea including the $M_{\mathrm{w}} 5.8$ earthquake on 8 January 2013. Proc Jpn Acad Ser B 89:438-445

Sarlis NV, Christopoulos S-RG (2012) Natural time analysis of the Centennial earthquake catalog. Chaos 22:023123

Sarlis NV, Skordas ES, Lazaridou MS, Varotsos PA (2008) Investigation of seismicity after the initiation of a seismic electric signal activity until the main shock. Proc Jpn Acad Ser B 84:331-343

Sarlis NV, Skordas ES, Varotsos PA (2009) Multiplicative cascades and seismicity in natural time. Phys Rev E 80:022102

Sarlis NV, Skordas ES, Varotsos PA (2010) Nonextensivity and natural time: the case of seismicity. Phys Rev E 82:021110

Sarlis NV, Skordas ES, Varotsos PA, Nagao T, Kamogawa M, Tanaka H, Uyeda S (2013) Minimum of the order parameter fluctuations of seismicity before major earthquakes in Japan. Proc Natl Acad Sci USA 110:13734-13738

Sarlis NV, Varotsos PA and Skordas ES (2014). Fluctuation theorem and natural time analysis, arXiv:1301.7634v7 [cond-mat.statmech] (7 Aug 2014)

Sarlis NV, Skordas ES, Varotsos PA, Nagao T, Kamogawa H, Uyeda S (2015) Spatiotemporal variations of seismicity before major earthquakes in the Japanese area and their relation with the epicentral locations. Proc Natl Acad Sci USA 112:986-989

Sornette D (2000) Critical phenomena in the natural sciences: chaos, fractals, selforganization, and disorder: concepts and tools. Springer, Berlin

Stanley HE (1999) Scaling, universality, and renormalization: three pillars of modern critical phenomena. Rev Mod Phys 71:S358S366
Telesca L (2010) Analysis of Italian seismicity by using a nonextensive approach. Tectonophysics 494:155-162

Telesca L, Lovallo M (2009) Non-uniform scaling features in central Italy seismicity: a non-linear approach in investigating seismic patterns and detection of possible earthquake precursors. Geophys Res Lett 36:L01308

Turcotte DL (1997) Fractals and Chaos in Geology and Geophysics. Cambridge University Press, Cambridge

Varotsos P (2005) The Physics of Seismic Electric Signals. Terrapub, Tokyo

Varotsos P, Alexopoulos K (1984a) Physical properties of the variations of the electric field of the earth preceding earthquakes, I. Tectonophysics 110:73-98

Varotsos P, Alexopoulos K (1984b) Physical properties of the variations of the electric field of the earth preceding earthquakes, II. Determination of epicenter and magnitude. Tectonophysics 110:99-125

Varotsos P, Alexopoulos K (1986) Thermodynamics of Point Defects and their Relation with Bulk Properties. North Holland, Amsterdam

Varotsos P, Lazaridou M (1991) Latest aspects of earthquake prediction in Greece based on seismic electric signals. Tectonophysics 188:321-347

Varotsos P, Alexopoulos K, Lazaridou M (1993) Latest aspects of earthquake prediction in Greece based on seismic electric signals, II. Tectonophysics 224:1-37

Varotsos PA, Sarlis NV, Skordas ES (2001) Spatio-temporal complexity aspects on the interrelation between seismic electric signals and seismicity. Pract Athens Acad 76:294-321

Varotsos PA, Sarlis NV, Skordas ES (2002a) Long range correlations in the signals that precede rupture. Phys Rev E 66:011902

Varotsos PA, Sarlis NV, Skordas ES (2002b) Seismic electric signals and seismicity: on a tentative interrelation between their spectral content. Acta Geophys Pol 50:337-354

Varotsos PA, Sarlis NV, Skordas ES (2003a) Attempt to distinguish electric signals of a dichotomous nature. Phys Rev E 68:031106

Varotsos PA, Sarlis NV, Skordas ES (2003b) Long-range correlations in the electric signals the precede rupture: further investigations. Phys Rev E 67:021109

Varotsos PA, Sarlis NV, Tanaka HK, Skordas ES (2005) Similarity of fluctuations in correlated systems: the case of seismicity. Phys Rev E 72:041103

Varotsos PA, Sarlis NV, Skordas ES, Lazaridou MS (2008) Fluctuations, under time reversal, of the natural time and the entropy distinguish similar looking electric signals of different dynamics. J Appl Phys 103:014906

Varotsos PA, Sarlis NV, Skordas ES (2009) Detrended fluctuation analysis of the magnetic and electric field variations that precede rupture. Chaos 19:023114

Varotsos PA, Sarlis NV, Skordas ES (2011a) Natural time analysis: the new view of time: precursory seismic electric signals, earthquakes and other complex time-series. Springer, Berlin

Varotsos PA, Sarlis NV, Skordas ES (2011b) Scale-specific order parameter fluctuations of seismicity in natural time before mainshocks. EPL 96:59002

Varotsos P, Sarlis NV, Skordas ES, Uyeda S, Kamogawa M (2011c) Natural time analysis of critical phenomena. Proc Natl Acad Sci USA 108:11361-11364

Varotsos PA, Sarlis NV, Skordas ES (2012) Scale-specific order parameter fluctuations of seismicity before mainshocks: natural time and detrended fluctuation analysis. EPL 99:59001

Varotsos PA, Sarlis NV, Skordas ES, Lazaridou MS (2013) Seismic electric signals: an additional fact showing their physical interconnection with seismicity. Tectonophysics 589:116-125

Varotsos PA, Sarlis NV, Skordas ES (2014) Study of the temporal correlations in the magnitude time series before major earthquakes in Japan. J Geophys Res Space Phys 119:9192-9206 